\title{
Clustered Planarity: Clusters with Few Outgoing Edges
}

\author{
Vít Jelínek^, Ondřej Suchý^, Marek Tesař, and Tomáš Vyskočil ${ }^{\star}$ \\ Department of Applied Mathematics \\ Charles University \\ Malostranské nám. 25, 11800 Praha, Czech Republic \\ \{jelinek, suchy, tesulo, tiger\}@kam.mff.cuni.cz
}

\begin{abstract}
We present a linear algorithm for c-planarity testing of clustered graphs, in which every cluster has at most four outgoing edges.
\end{abstract}

\section{Introduction}

Clustered planarity is one of the challenges of contemporary Graph Drawing. It arises naturally when we want to draw the graph with further constraints on embedding of the vertices. This includes for example visualizing a computer network with the computers of the same department, faculty and institution being grouped together. Another application is in designing an integrated circuit with the connectors of each components being close to each other and the logical parts of the circuit being grouped together. There are many other applications including visualizations of process interaction, social networks etc.

The concept of the clustered graph - a graph equipped with a system of subsets of vertices (called clusters), that can be recursive - was first introduced by Feng et al. in [7. In the same paper they also proved that clustered planarity (shortly c-planarity) can be tested in polynomial time for c-connected clustered graphs (where each cluster induces a connected subgraph of the underlying graph). This was later improved by Dahlhaus [4 to a linear time algorithm. The paper [7] also contains a useful characterization of the c-planar graphs: Graph is c-planar if and only if there is a set of edges (usually called a saturator) that can be added to this graph to obtain a c-connected c-planar clustered graph.

Since then many algorithms for testing the c-planarity were based on searching for a saturator. These include an $O\left(n^{2}\right)$-time algorithm for "almost" c-connected clustered graphs by Gutwenger et al. in 9010. An efficient algorithm for clusters with cyclic structure on a cycle was developed in [3. The case of disjoint clusters on an embedded graph with small faces was recently addressed in [5]. Very similar result was at the same time independently published by Jelínková et al. 12. The paper 12] also contains an $O\left(n^{3}\right)$-time algorithm for clusters of size at most three on a rib-Eulerian graph. This is an Eulerian graph that is obtained from a constant size 3-connected graph by multiplying and then subdividing edges.

\footnotetext{
^ Supported by grant 201/05/H014 of the Czech Science Foundation.
} 
Another approach is to mimic the original proof of Feng et al. [7] where the behavior of the connected clusters is described by special trees. In this way a slight generalization to extrovert clustered graphs was given by Goodrich et al. 8]. In an extrovert clustered graph the parent cluster of any disconnected cluster is connected and every component of any disconnected cluster is incident to an edge which leads outside of its parent cluster.

We should also mention that every c-planar graph can be drawn by straight lines with clusters represented by convex polygons [6]. Another interesting contribution is the characterization of completely connected clustered graphs (where each subgraph induced by a cluster and its complement are connected) 1]: A completely connected clustered graph is c-planar if and only if the underlying graph is planar. More results on c-planarity can be found in 2. Despite the number of results the complexity of testing the c-planarity for general instances remains open.

In this paper we focus on the situation where the number of outgoing edges of each cluster is small. We notice that in this case the behavior of the clusters can be simulated by special graphs, no matter whether the subgraph induced by the cluster is connected or not. We use these ideas to develop a linear time algorithm to test such graphs for c-planarity. As far as we know this is the first algorithm that can be used in the cases where the underlying graph is not connected at all or has very few edges in total. In particular we prove the following theorem:

Theorem 1. Clustered planarity can be decided in linear time for instances, where each cluster has at most 4 outgoing edges.

Section 2 is devoted to the basic definitions. We also show there that if there is a cluster with no outgoing edges, then the instance could be split into an instance formed by the subclusters of the cluster and one formed by the rest. In Section 3 we show how to replace the clusters by special graphs with the same behavior and prove that this does not affect the c-planarity. The algorithm is described in Section 4, together with the proofs of the correctness and the running time. In Section [5] we show that the approach cannot be generalized this way to the case of five or more outgoing edges.

\section{Preliminaries}

Let $S_{r}$ denote the set of all permutations of the set $\{1,2, \ldots, r\}$. A permutation $\pi \in S_{r}$ is represented by $r$-tuple $(\pi(1) \ldots \pi(r))$.

Regarding the graph notations, we follow the standard notation on finite loopless graphs. A graph is an ordered pair $G=(V, E)$, where $V$ is the set of vertices and $E$ is the set of edges i.e. pairs of vertices. We simply write $u v$ instead of $\{u, v\}$ for edges. If $U \subseteq V$, then $G[U]$ is the induced subgraph of $G$ on vertices $U$ and $G \backslash U=G[V \backslash U]$. Let $n$ denote the number of vertices $|V|$ of the graph $G$.

A cluster set on the graph $G=(V, E)$ is a set $\mathcal{C} \subseteq \mathcal{P}(V(G))$ such that for all $C, D \in \mathcal{C}$, either $C$ and $D$ are disjoint or they are in inclusion; the pair $(G, \mathcal{C})$ is called a clustered graph. The elements of $\mathcal{C}$ are called clusters. A clustered 
planar embedding of $(G, \mathcal{C})$ is a planar embedding $e m b$ of $G$ together with a mapping $e m b_{c}$ that assigns to every cluster $C \in \mathcal{C}$ a planar region $e m b_{c}(C)$ whose boundary is a closed Jordan curve and such that

- for each vertex $v \in V$ and every cluster $C \in \mathcal{C}$, it holds that $\operatorname{emb}(v) \in$ $e m b_{c}(C)$ if and only if $v \in C$,

- for every two clusters $C$ and $D$, the regions $e m b_{c}(C)$ and $e m b_{c}(D)$ are disjoint (in inclusion) if and only if $C$ and $D$ are disjoint (in inclusion, respectively), and

- for every edge $e \in E$ and every cluster $C \in \mathcal{C}$ the curve $e m b(e)$ crosses the boundary of $e m b_{c}(C)$ at most once.

A clustered graph is called clustered planar (shortly c-planar) if it allows a clustered planar embedding.

The following observation is a trivial consequence of the definition:

Remark 1. A pair $(G, \emptyset)$ is c-planar if and only if the graph $G$ is planar.

We say that $C \in \mathcal{C}$ is a cluster of the bottom-most level if there is no $C^{\prime} \in \mathcal{C}$ such that $C^{\prime} \subset C$.

An edge $e=u v$ is an outgoing edge of a cluster $C$ if $u \in C, v \in V \backslash C$ or vice versa 1 Let $r(C)=|\{e=u v \mid e \in E, u \in C, v \in V \backslash C\}|$ denote the number of outgoing edges of a cluster $C$. If the cluster is clear from context we will just use notation $r$ instead of $r(C)$.

Lemma 1. If $C$ has no outgoing edges then $(G, \mathcal{C})$ is c-planar if and only if $\left(G \backslash C, \mathcal{C}_{1}\right)$ and $\left(G[C], \mathcal{C}_{2}\right)$ are c-planar, where $\mathcal{C}_{1}=\{A \backslash C \mid A \in \mathcal{C}, A \neq C, A \supset$ $C\} \cup\{A \mid A \in \mathcal{C}, A \cap C=\emptyset\}$ and $\mathcal{C}_{2}=\{B \mid B \in \mathcal{C}, B \neq C, B \subset C\}$.

Proof. The direction from left to right is easy, we just omit from the embedding the parts that are no longer necessary.

So suppose that we have a c-planar embedding $e m b_{1}$ of $\left(G \backslash C, \mathcal{C}_{1}\right)$ and a c-planar embedding $e m b_{2}$ of $\left(G[C], \mathcal{C}_{2}\right)$. Take an arbitrary point $x$ in the plane, such that for all clusters $A \in \mathcal{C}_{1}$ the following holds: $x$ lies inside the region $\left(e m b_{1}\right)_{c}(A)$ if and only if $C \subseteq A$. Suppose that there is neither vertex nor edge of $G \backslash C$ nor border of a cluster of $\mathcal{C}_{1}$ in distance less than $\epsilon$ from $x$ in $e m b_{1}$. Now shrink the embedding $e m b_{2}$ so that it fits into the $\frac{\epsilon}{2}$-disc centered in $x$. Then take this disc as the embedding of $C$.

It is easy to check that we obtain a c-planar embedding of $(G, \mathcal{C})$, since the embeddings $e m b_{1}$ and $e m b_{2}$ cross neither each other nor the embedding of $C$, the inclusions of the clusters are preserved and the embedding of the cluster $C$ contains exactly the embedding of the vertices, edges and clusters it should contain.

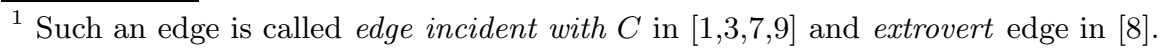



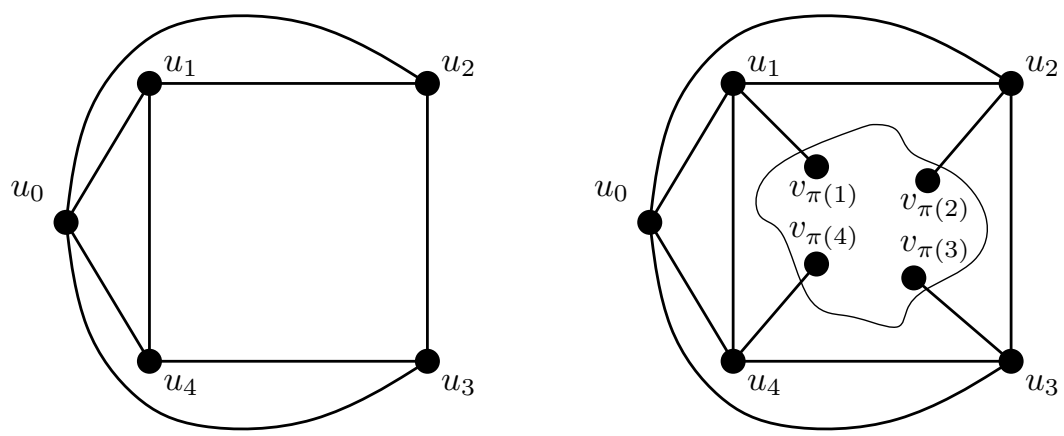

Fig. 1. The test graph $\mathrm{T}$ and the graph $T_{C}^{\pi}$ from Definition 1

\section{Replacement of Clusters by Graphs}

Through this section we suppose, that we have some fixed cluster $C \in \mathcal{C}$ of bottom-most level, that has at most 4 outgoing edges. Having Lemma 1 in hand we assume that $1 \leq r=r(C) \leq 4$.

We denote the outgoing edges by $\left\{e_{1}, \ldots, e_{r}\right\}$. We also suppose that $e_{i}=v_{i} w_{i}$ for all $i$, where $v_{i} \in C$ and $w_{i} \in V \backslash C$ (maybe $w_{i}=w_{j}$ or $v_{i}=v_{j}$ for some $i \neq j)$.

We denote by $T$ the following test graph $T=\left(\left\{u_{0}, u_{1}, u_{2}, u_{3}, u_{4}\right\},\left\{u_{0} u_{1}, u_{0} u_{2}\right.\right.$, $\left.u_{0} u_{3}, u_{0} u_{4}, u_{1} u_{2}, u_{2} u_{3}, u_{3} u_{4}, u_{4} u_{1}\right\}$ ) (see Fig. 1).

Definition 1. We say that the cluster $C$ admits a permutation $\pi \in S_{r}$ if and only if the graph $T_{C}^{\pi}$ created from $T \cup G[C]$ by adding edges $u_{i} v_{\pi(i)}, 1 \leq i \leq r$ is planar.

Lemma 2. If the cluster $C$ admits the permutation $\pi \in S_{r}$ then there exists a planar embedding of the graph $T_{C}^{\pi}$ such that the vertices of $C$ are embedded inside and the vertex $u_{0}$ outside the cycle $u_{1}, \ldots, u_{4}, u_{1}$ of $T$. Moreover we can prescribe this cycle to be oriented clockwise in the embedding.

Proof. First we take some planar embedding of the graph $T_{C}^{\pi}$. Now we take the edges incident with $u_{0}$ in the clockwise order $u_{0} u_{i_{1}}, u_{0} u_{i_{2}}, u_{0} u_{i_{3}}, u_{0} u_{i_{4}}$. For every $u_{0} u_{i}$ and $u_{0} u_{j}$ two consecutive of them (either $\{i, j\}=\left\{i_{k}, i_{k+1}\right\}$ for some $k=1,2$ or 3 or $\left.\{i, j\}=\left\{i_{1}, i_{4}\right\}\right)$ we can draw a new curve from $u_{i}$ to $u_{j}$ along the curve $u_{i} u_{0}$ and then $u_{0} u_{j}$ so that it does not cross any other edge and area surrounded by the curves $u_{i} u_{0}, u_{0} u_{j}$ and the new curve contains no vertex (see Fig. 2).

Suppose for a contradiction that some of the newly drawn curves connects two non-adjacent vertices, for example $u_{1}$ and $u_{3}$ (the case of $u_{2}$ and $u_{4}$ being similar). Since the new curves connect $u_{1}$ to at most one of the vertices $u_{2}$ and $u_{4}$ and we drew two curves from each $u_{i}$, we also connected $u_{2}$ and $u_{4}$. But this means that the newly drawn curves together with the original edges form 

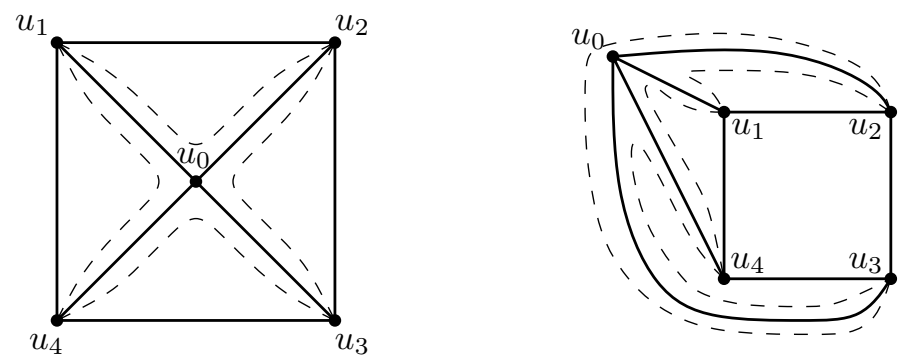

Fig. 2. Situation from the proof of Lemma 2

a planar embedding of $K_{5}$, which is a contradiction. So we know that all the curves that we drew newly connect two already adjacent vertices of the cycle.

Now we take these newly drawn curves as the embedding of the edges of the cycle. Then there is just $u_{0}$ inside the cycle and it remains to change the outer face to one of the newly obtained empty triangles, such that the vertex $u_{0}$ will be on the boundary of the outer face.

If the cycle is embedded in wrong direction, then we take the axis symmetry of the embedding.

Lemma 3. If $(G, \mathcal{C})$ is c-planar, then $C$ admits some permutation.

Proof. We suppose that $(G, \mathcal{C})$ is c-planar and we fix a planar embedding $e m b$. Let $f$ be the boundary of $\operatorname{emb}_{C}(C)$ (so $f$ is a closed Jordan curve). Now we can start in an arbitrary point of this curve and move along this curve in the clockwise direction and we cross the edges $e_{1}, e_{2}, \ldots, e_{r}$ in some order $e_{i_{1}}, e_{i_{2}}, \ldots, e_{i_{r}}$. Denote the crossing points as $P_{1}, P_{2}, \ldots, P_{r}$ (in the same order). If $r<4$ then we can choose new points $P_{r+1}, \ldots, P_{4}$ in such a way, that we meet the points $P_{1}, \ldots, P_{4}$ in this order when we move along the curve $f$ in the clockwise direction and all these points are distinct.

Now we consider the planar embedding $e m b^{\prime}$ of $G[C]$ which corresponds to the embedding $e m b$ of the graph $G$, place new vertices $u_{1}, \ldots, u_{4}$ to the points $P_{1}, \ldots, P_{4}$ and a vertex $u_{0}$ outside of the region bounded by the curve $f$. Clearly we can add edges $\left(u_{1}, v_{i_{1}}\right), \ldots,\left(u_{r}, v_{i_{r}}\right)$ and embed these edges on curves which corresponded to edges $e_{1}, \ldots, e_{r}$ inside of the region $\mathrm{emb}_{C}(C)$ and we can also add edges $\left(u_{1}, u_{2}\right),\left(u_{2}, u_{3}\right),\left(u_{3}, u_{4}\right)$ and $\left(u_{4}, u_{1}\right)$ and embed them on the curve $f$ in such a way that these edges may intersect only in vertices $u_{1}, u_{2}, u_{3}$ or $u_{4}$. It is clear that we can add edges $\left(u_{0}, u_{1}\right), \ldots,\left(u_{0}, u_{4}\right)$ and embed them in such a way that these edges will be outside of the region bounded by $f$ and every two edges will cross only in the vertex $u_{0}$.

This way we obtain a planar embedding of the graph $T_{C}^{\pi}$ where $\pi=\left(a_{i_{1}} \ldots a_{i_{r}}\right)$. Thus $C$ admits the permutation $\pi$.

Lemma 4. If the cluster $C$ admits a permutation $\pi=\left(a_{1} a_{2} \ldots a_{r}\right)$ then it also admits permutations $\left(a_{r} a_{1} \ldots a_{r-1}\right)$ and $\left(a_{r} a_{r-1} \ldots a_{1}\right)$. 
Proof. We obtain the planar embedding of $T_{C}^{\delta}, \delta=\left(a_{r} a_{1} \ldots a_{r-1}\right)$ from the planar embedding of $T_{C}^{\pi}$ simply by relabeling the vertices such that $u_{1}$ becomes $u_{2}, u_{2}$ becomes $u_{3}, u_{3}$ becomes $u_{4}$ and $u_{4}$ becomes $u_{1}$ and if $r<4$ then it is necessary to replace the edge $v_{a_{r}} u_{r+1}$ by a new edge $v_{a_{r}} u_{1}$ which goes along the edges $v_{a_{r}} u_{r+1}, u_{r+1} u_{r+2}, \ldots, u_{4} u_{1}$ such that it doesn't cross any other edge.

For $r \geq 3$ the second part can be done similarly - it is enough to relabel such that $u_{1}$ becomes $u_{3}$ and $u_{3}$ becomes $u_{1}$ and if $r=4$ then we use the first part to achieve permutation $\left(a_{r} a_{r-1} \ldots a_{1}\right)$. For $r<3$ the first part also proves the second part.

We can now define a relation $\sim^{\prime}$ on the permutations from the set $S_{r}$ by $\left(a_{1} a_{2} \ldots a_{r}\right) \sim^{\prime}\left(a_{r} a_{1} \ldots a_{r-1}\right)$ and $\left(a_{1} a_{2} \ldots a_{r}\right) \sim^{\prime}\left(a_{r} a_{r-1} \ldots a_{1}\right)$. If we take $\sim$ to be the transitive closure of $\sim^{\prime}$, then it is easy to show that $\sim$ is also reflexive and symetric. Thus $\sim$ is an equivalence. We will sometimes call the equivalence classes of this equivalence circular permutations The sets $S_{1}, S_{2}, S_{3}$ have just one equivalence class under $\sim$ while the set $S_{4}$ is partitioned into following three equivalence classes (they can be distinguished by the number that is "opposite" to the number 1$)$ :

$$
\begin{aligned}
& S_{4}^{2}=\{(1324),(3241),(2413),(4132),(4231),(1423),(3142),(2314)\}, \\
& S_{4}^{3}=\{(1234),(2341),(3412),(4123),(4321),(1432),(2143),(3214)\}, \\
& S_{4}^{4}=\{(1243),(2431),(4312),(3124),(3421),(1342),(2134),(4213)\} .
\end{aligned}
$$

Definition 2. We define the corresponding graph for cluster $C$ as follows (see Fig (3).

1. If $r \leq 3$ and $C$ admits some permutation, then the corresponding graph for $C$ is $R_{r}$.

2. If there is a labeling of the outgoing edges such that $C$ admits permutations from $S_{4}^{2}, S_{4}^{3}$, $S_{4}^{4}$ then the corresponding graph for $C$ with this labeling is $R_{4}^{234}$.

3. If there is a labeling of the outgoing edges such that $C$ admits a permutation from $S_{4}^{2}$ and from $S_{4}^{3}$, but no permutation from $S_{4}^{4}$ then the corresponding graph for $C$ with this labeling is $R_{4}^{23}$.

4. If there is a labeling of the outgoing edges such that $C$ admits a permutation from $S_{4}^{2}$, but no permutation from $S_{4}^{3} \cup S_{4}^{4}$ then the corresponding graph for $C$ with this labeling is $R_{4}^{2}$.

Clearly, if $r \leq 3$ then the cluster $C$ has unique corresponding graph. Since the sets $S_{4}^{2}, S_{4}^{3}$, and $S_{4}^{4}$ form a decomposition of $S_{4}$, from Lemma 4 we know that the cluster $C$ admits all permutations from some non-empty combination of sets $S_{4}^{2}, S_{4}^{3}$, and $S_{4}^{4}$.

If the cluster $C$ admits just permutations from the set $S_{4}^{i}$ then by relabeling of edge $e_{2}$ by $e_{i}$ and incident vertices $v_{2}$ by $v_{i}$ and $w_{2}$ by $w_{i}$ (if $i=2$ we don't need to do it) we get labeling of the cluster $C$ which admits only permutations from the set $S_{4}^{2}$. So the cluster $C$ has unique corresponding graph. 


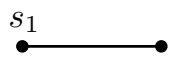

$R_{1}$

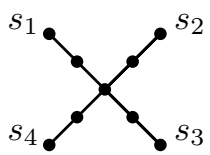

$R_{4}^{2,3,4}$

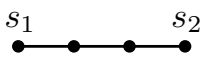

$R_{2}$

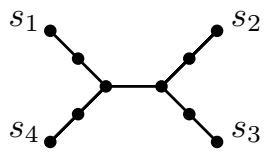

$R_{4}^{2,3}$

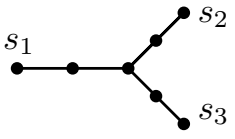

$R_{3}$

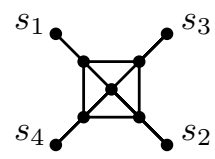

$R_{4}^{2}$

Fig. 3. The graphs $R_{1}, R_{2}, R_{3}, R_{4}^{234}, R_{4}^{23}$ and $R_{4}^{2}$

If the cluster $C$ admits just permutations from two distinct sets $S_{4}^{i}$ and $S_{4}^{j}$ then we make similar relabeling of outgoing edges and incident vertices such that resulting relabeling makes the cluster $C$ admit just permutations from the sets $S_{4}^{2}$ and $S_{4}^{3}$ and the cluster $C$ has unique corresponding graph.

As a consequence we get the following corollary.

Corollary 1. If $C$ admits a permutation then there is a labeling of outgoing edges of $C$ such that $C$ has a corresponding graph with this labeling.

For the rest of the paper we will use this new labeling.

Definition 3. Let $C$ be a cluster of the bottom-most level with outgoing edges $e_{1}, \ldots, e_{r}$ where $1 \leq r \leq 4, e_{i}=v_{i} w_{i}$ for all $i$, where $v_{i} \in C$ and $w_{i} \in V \backslash C$. Let $R$ be a corresponding graph to the cluster $C$ in this labeling. Then a replacement of cluster $C$ by a corresponding graph $R$ in $(G, \mathcal{C})$ is a clustered graph $\left(G^{\prime}, \mathcal{C}^{\prime}\right)$ such that $G^{\prime}$ is created from $(G \backslash C) \cup R$ by unification of $w_{1}, \ldots, w_{r}$ with $s_{1}, \ldots, s_{r}$ (respectively) and $\mathcal{C}^{\prime}$ is created from $\mathcal{C} \backslash\{C\}$ by replacing every $C^{\prime} \supseteq C$ by $\left(C^{\prime} \backslash C\right) \cup\left(V(R) \backslash\left\{s_{1}, \ldots, s_{r}\right\}\right)$.

Proposition 1. Let $\left(G^{\prime}, \mathcal{C}^{\prime}\right)$ be the replacement of cluster $C$ by a corresponding graph $R$. Then $(G, \mathcal{C})$ is c-planar if and only if $\left(G^{\prime}, \mathcal{C}^{\prime}\right)$ is c-planar.

Proof. (" $\Rightarrow ":)$ We suppose that $(G, \mathcal{C})$ is c-planar and we fix some planar embedding $e m b$. Without loss of generality we can suppose that $e m b_{\mathcal{C}}(C)$ is a disc (because this region is homeomorphic to a disc). Suppose that the edges $e_{1}, \ldots, e_{r}$ cross the boundary of $e m b_{\mathcal{C}}(C)$ in (clockwise) order $e_{i_{1}}, \ldots, e_{i_{r}}$ and without loss of generality $i_{1}=1$.

If $r<4$ then we simply remove cluster $C$ with edges $e_{1}, \ldots, e_{r}$ and draw the graph $R_{r}$ corresponding to $C$ in a such way, that we identify vertex $s_{i}$ with $w_{i}$ for all $i \in\{1, \ldots, r\}$ and all other vertices of $R_{r}$ draw inside $e m b_{\mathcal{C}}(C)$ in such a way, that edges of $R_{r}$ don't cross any other edge of original graph nor other edge of $R_{r}$. This is clearly possible, it is enough to draw the edges outside the 


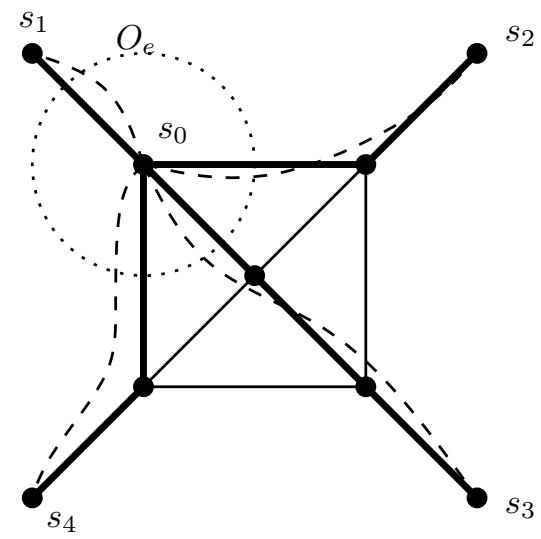

Fig. 4. Situation from the proof of Proposition 1 (part " $\Leftarrow ")$

disc $e m b_{\mathcal{C}}(C)$ along the deleted edges $e_{1}, \ldots, e_{r}$ and inside $e m b_{\mathcal{C}}(C)$ we can draw edges (or parts of edges) as noncrossing segments. This embedding of $G^{\prime}$ shows that $\left(G^{\prime}, \mathcal{C}^{\prime}\right)$ is c-planar.

If the corresponding graph for $C$ is $R_{4}^{234}$ then we can construct a c-planar embedding of $C^{\prime}$ in the same way as for $r<4$.

If the corresponding graph for $C$ is $R_{4}^{2}$ then the ordered set $\left(i_{1}, i_{2}, i_{3}, i_{4}\right)$ must be equal to $(1,3,2,4)$ or $(1,4,2,3)$ because $C$ admits only permutations from $S_{4}^{2}$ (otherwise we could find a permutation $\pi \notin S_{4}^{2}$ such that $T_{C}^{\pi}$ is planar which is a contradiction). Now we delete the cluster $C$ and add the graph $R_{4}^{2}$ in such a way that all the vertices of $R_{4}^{2}$ will be inside the $\operatorname{disc} e m b_{\mathcal{C}}(C)$ and we identify vertices $s_{i}$ with $w_{i}$ for all $i \in\{1, \ldots, 4\}$ and any edge of $R_{4}^{2}$ will not cross any original edge nor any new edge of $R_{4}^{2}$. This is also clearly possible, it is enough to draw the edges outside the $\operatorname{disc} e m b_{\mathcal{C}}(C)$ along the deleted edges $e_{1}, \ldots, e_{4}$ and inside $e m b_{\mathcal{C}}(C)$ we can draw the edges (or parts of the edges) as noncrossing segments. This embedding of $G^{\prime}$ shows that $\left(G^{\prime}, \mathcal{C}^{\prime}\right)$ is c-planar.

If the corresponding graph for cluster $C$ is $R_{4}^{23}$ then we continue similarly as in the previous cases. The ordered set $\left(i_{1}, i_{2}, i_{3}, i_{4}\right)$ must be equal to $(1,3,2,4)$, $(1,4,2,3),(1,2,3,4)$ or $(1,4,3,2)$ so again it is easy to replace the vertices and the edges of $C$ by the graph $R_{4}^{23}$ by identifying the vertices $s_{i}$ with $w_{i}$ for all $i \in\{1, \ldots, 4\}$ which proves that $\left(G^{\prime}, \mathcal{C}^{\prime}\right)$ is c-planar again.

$(" \Leftarrow ":)$ Suppose we have a c-planar embedding of $\left(G^{\prime}, \mathcal{C}^{\prime}\right)$. Moreover suppose that in the case $R=R_{4}^{2}$ there is nothing embedded in any interior face of $R$. This can be easily achieved in a similar way as in the proof of Lemma 2 . We take an arbitrary spanning tree of the graph $R$ and let $s_{0}$ denote its arbitrary vertex different from $s_{1}, \ldots, s_{r}$. Now draw the $r$ curves connecting $s_{0}$ to $s_{1}, s_{2}, \ldots, s_{r}$ along the unique paths connecting the vertices in the tree, so that they do not cross each other nor anything in the embedding, except possibly for the edges of $R$. Then remove the original edges of $R$.

Now take some $\epsilon$ such that there are no edges, vertices nor clusters embedded in distance less than $\epsilon$ from $s_{0}$, except for the curves incident with $s_{0}$. Denote 
by $O_{\epsilon}$ the circle of radius $\epsilon$ with center $s_{0}$. Suppose $P_{i}$ is the last intersection of the curve $s_{0} s_{i}$ with $O_{\epsilon}$. We can assume, that $\epsilon$ is so small, that if we label these curves clockwise $s_{0} s_{i_{1}}, s_{0} s_{i_{2}}, \ldots, s_{0} s_{i_{r}}$ as they leave $s_{0}$, then $P_{i_{1}}, P_{i_{2}}, \ldots, P_{i_{r}}$ are the points $P_{i}$ in the clockwise order along $O_{\epsilon}$. (We can assume, that each curve in the embedding is formed by finitely many straight line segments and circular arcs.)

By case analysis we show, that $C$ admits the permutation $\pi=\left(i_{1} i_{2} \ldots i_{r}\right)$. This is clear if $R=R_{1}, R_{2}, R_{3}$ or $R_{4}^{234}$. The graph $R_{4}^{2} \backslash\left\{s_{1}, \ldots, s_{4}\right\}$ is 3-connected so the order of the edges is given in this case (up to the equivalence $\sim$ ) and the permutation $\pi$ is in $S_{4}^{2}$. If $R=R_{4}^{23}$ and $\pi \in S_{4}^{4}$, then by connecting the neigbouring edges we obtain a planar embedding of $K_{3,3}-$ a contradiction.

So we take the planar embedding of $T_{C}^{\pi}$ guaranteed by Lemma 2 and remove the vertex $u_{0}$. We can take a homeomorphic copy of this embedding of $T_{C}^{\pi} \backslash$ $\left\{u_{0}\right\}$, in which the cycle $u_{1}, u_{2}, u_{3}, u_{4}, u_{1}$ coincides with a circle $O_{\epsilon}$ and the vertices $u_{1}, u_{2}, \ldots, u_{r}$ are embedded at the points $P_{i_{1}}, P_{i_{2}}, \ldots, P_{i_{r}}$, respectively. We replace the interior of $O_{\epsilon}$ by such an embedding.

We are ready to describe an embedding of $(G, \mathcal{C})$. For every $i$ the concatenation of the curve $v_{\pi(i)} u_{i}=P_{\pi(i)}$ and $P_{\pi(i)} s_{\pi(i)}$ forms an embedding of the edge $v_{\pi(i)} w_{\pi(i)}$ that crosses no other edge of $G^{\prime}$ or $G[C]$. Moreover, it crosses the boundary of each cluster of $C^{\prime}$ at most once, since there were no cluster boundaries inside $O_{\epsilon}$, curve $P_{\pi(i)} s_{\pi(i)}$ was drawn along some edges of $R$ and among them only the one incident with $s_{\pi(i)}$ could cross some cluster boundary and also at most once, because we started with a c-planar embedding of $\left(G^{\prime}, \mathcal{C}^{\prime}\right)$. It remains to take $O_{\epsilon}$ as the boundary of the cluster $C$. It only crosses the edges $w_{i} v_{i}$. Furthermore, since curve $s_{i} P_{i}$ (recall that $s_{i}=w_{i}$ ) lies completely outside $O_{\epsilon}$ (except for $P_{i}$ ), while $P_{i} v_{i}$ lies completely inside $O_{\epsilon}$ (except for $P_{i}$ ), $O_{\epsilon}$ crosses the edge $w_{i} v_{i}$ exactly once (in the point $P_{i}$ ). There are no other crossings, since they would have to be in the original c-planar embedding of $\left(G^{\prime}, C^{\prime}\right)$ too.

\section{The Algorithm}

The algorithm is described in Fig. 5 ,

Proposition 2. The algorithm correctly decides c-planarity for instances, where each cluster has at most 4 outgoing edges.

Proof. We first prove by the mathematical induction that for every $0 \leq i \leq|\mathcal{C}|$, the pair $\left(G_{i}, C_{i}\right)$ is defined and c-planar if and only if $(G, \mathcal{C})$ is c-planar. This is certainly true for $i=0$. Now suppose that this is true for every $i^{\prime}<i$ and let us prove it for $i$.

In the case $r(C)=0$ we have two possibilities. Either $G_{i-1}[C]$ is not planar, then also $G$ is not planar and $\left(G_{i-1}, \mathcal{C}_{i-1}\right)$ is definitely not c-planar. Then the algorithm correctly rejects (and $G_{j}, \mathcal{C}_{j}$ is not defined for $j \geq i$ ). Or $G_{i-1}[C]$ is planar and by Lemma 1 and Remark 1 pair $\left(G_{i-1}, \mathcal{C}_{i-1}\right)$ is c-planar if and only 
Input: Graph $G$ and cluster set $\mathcal{C}$, where each cluster has at most 4 outgoing edges.

Task: Accept $(G, C)$ if and only if $(G, C)$ is clustered planar.

1. Set $G_{0}:=G, \mathcal{C}_{0}:=\mathcal{C}$.

2. For $i:=1$ to $|\mathcal{C}|$ do:

(a) Let $C$ be some cluster on the bottom-most level in $\mathcal{C}_{i-1}$.

(b) If $r(C)=0$ then

i. If $G_{i-1}[C]$ is planar then set

$G_{i}:=G_{i-1} \backslash C$

$\mathcal{C}_{i}:=\left\{A \backslash C \mid A \in \mathcal{C}_{i-1} \backslash\{C\}, A \supseteq C\right\} \cup\left\{A \mid A \in \mathcal{C}_{i-1}, A \nsupseteq C\right\}$

ii. else REJECT.

(c) else

i. For each permutation $\pi \in S_{r(C)}$ test whether $C$ admits $\pi$ (whether $T_{C}^{\pi}$ is planar)

ii. If $C$ admits no permutation, then REJECT.

iii. Let $\left(G_{i}, \mathcal{C}_{i}\right)$ be the replacement of cluster $C$ by the corresponding graph in $\left(G_{i-1}, \mathcal{C}_{i-1}\right)$.

3. If $G_{|\mathcal{C}|}$ is planar then ACCEPT, otherwise REJECT $\left(\mathcal{C}_{|\mathcal{C}|}=\emptyset\right)$.

Fig. 5. An overview of the algorithm

if $\left(G_{i}, \mathcal{C}_{i}\right)$ is, since $\{B \mid B \in \mathcal{C} \backslash\{C\}, B \subseteq C\}$ is empty (C is on the bottom-most level).

Now consider the case $1 \leq r(C) \leq 4$. If $C$ admits no permutation, then by Lemma 3 the pair $\left(G_{i-1}, \mathcal{C}_{i-1}\right)$ is not c-planar and the algorithm correctly rejects (and does not define $G_{j}, \mathcal{C}_{j}$ for $j \geq i$ ). Otherwise $C$ has a corresponding graph by Corollary 1 and from the Proposition 1 we know that $\left(G_{i-1}, \mathcal{C}_{i-1}\right)$ is c-planar if and only if $\left(G_{i}, \mathcal{C}_{i}\right)$ is c-planar.

Since $\left|\mathcal{C}_{i}\right|=\left|\mathcal{C}_{i-1}\right|-1$ whenever defined, we have $\left|\mathcal{C}_{|\mathcal{C}|}\right|=0$ and thus $\mathcal{C}_{|\mathcal{C}|}=\emptyset$ if $\mathcal{C}_{|\mathcal{C}|}$ is defined. But then $\left(G_{|\mathcal{C}|}, \mathcal{C}_{|\mathcal{C}|}\right)=\left(G_{|\mathcal{C}|}, \emptyset\right)$ is c-planar if and only if $G_{|\mathcal{C}|}$ is planar due to Remark 1, which completes the proof.

Proposition 3. The algorithm works in time $O(n)$.

Proof. The cycle is executed at most $|\mathcal{C}|$ times, in each time we delete one cluster or reject. When we omit a planarity testing, complexity of each step of cycle in the algorithm is bounded by constant. We add constant number of vertices and if we have a suitable representation of clusters (for example tree representation) we can find cluster on the bottom-most level in constant time too. And then for these operations we need $|\mathcal{C}|$ in complexity time. The algorithm touches each vertex at most three times, when we add, test, and remove it. For vertices which we added later we paid before, by constant in each iteration. And for the original vertices we need extra $n$ for planarity testing. Each vertex from the original graph we touch only once, because if we touch it we remove it or reject whole graph. Since $|\mathcal{C}|$ is bounded by $O(n)$, the complexity of our algorithm is $O(n)$. 


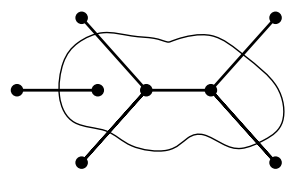

$D_{1}$

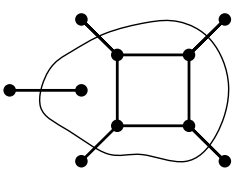

$D_{2}$

Fig. 6. Two clusters with 5 outgoing edges that cannot be represented by any connected graph

\section{The Limits of the Approach}

Let us consider clusters with more than 4 outgoing edges. Definition 11. Lemmas 2 and 4 easily generalize to this case as well as Lemma 3 . The problem with the generalization is that there are disconnected clusters with 5 outgoing edges that admit a combination of permutations which cannot be represented by a connected graph. In particular it can be shown that the two clusters from Fig. [6 have this property.

Let us try to formalize the result. Consider a graph $R$ that is supposed to be corresponding to some cluster $C$. Hence it has some distinguished vertices $s_{1}, \ldots, s_{r}$ of degree 1 that are supposed to be identified with the vertices of $G \backslash C$ when the cluster $C$ is replaced by $R$ in a graph $G$. Let $R^{\prime}=V(R) \backslash\left\{s_{1}, \ldots, s_{r}\right\}$. We say that the graph $R$ admits a permutation $\pi$, if the cluster $R^{\prime}$ of the clustered graph $\left(R,\left\{R^{\prime}\right\}\right)$ admits a permutation $\pi$.

Proposition 4. There is no connected graph that admits the same number of permutations as the cluster $D_{1}$ from Fig. 6.

Proof. We will count the circular permutations. In total there are 12 circular permutations on 5 elements, each representing 10 (standard) permutations. Observe first that $D_{1}$ admits 8 circular permutations. Now assume for a contradiction that there is a connected graph $R$ (with distinguished vertices $s_{1}, \ldots, s_{5}$ ) that admits also 8 circular permutations.

We observe that whenever we take a subgraph $Q$ of the graph $R, s_{1}, \ldots, s_{5} \in$ $V(Q)$, then the graph $Q$ admits at least the same number of permutations, since we can just ommit the unnecessary parts from the appropriate embedding. Now consider a subtree $T$ of $R$ with leaves of $T$ being exactly the vertices $s_{1}, \ldots, s_{5}$. It is clear that $R$ has such a subgraph since $R$ is connected.

Since $T$ has 5 leaves, it has at most 3 vertices of degree at least 3 - either it has 3 vertices of degree 3 , or one of degree 3 and one of degree 4 , or just one of degree 5 . It is not hard to check that in the first two cases $T$ admits 4 and 6 circular permutations, respectively. Thus in this cases $R$ cannot admit 8 circular permutations. The tree with just one vertex of degree 5 (among the vertices of degree at least 3 ) admits all 12 circular permutations. Thus we know that $T$ must be some subdivision of $K_{1,5}$. 
If $R$ contains no path connecting two different branches of $T$, then clearly $R$ admits the same permutations as $T$ i.e. all 12 circular permutations. On the other hand, if $R$ contains a path between two branches of $T$, then there is another tree $T^{\prime}$, subgraph of $R$, that has one vertex of degree 4 and one vertex of degree 3 . But this means that $R$ admits at most 6 permutations - a contradiction.

\section{References}

1. Cornelsen, S., Wagner, D.: Completely connected clustered graphs. Journal of Discrete Algorithms 4(2), 313-323 (2006)

2. Cortese, P.F., Di Battista, G.: Clustered planarity. In: ACM SoCG 2005, pp. 32-34 (2005)

3. Cortese, P.F., Di Battista, G., Patrignani, M., Pizzonia, M.: Clustering cycles into cycles of clusters. Journal of Graph Algorithms and Applications 9(3), 391-413 (2005); In: Pach, J. (ed.) GD 2004. LNCS, vol. 3383, pp. 391-413. Springer, Heidelberg (2005)

4. Dahlhaus, E.: A linear time algorithm to recognize clustered planar graphs and its parallelization. In: Lucchesi, C.L., Moura, A.V. (eds.) LATIN 1998. LNCS, vol. 1380, pp. 239-248. Springer, Heidelberg (1998)

5. Di Battista, G., Frati, F.: Efficient C-planarity testing for embedded flat clustered graphs with small faces. In: Hong, S.-H., Nishizeki, T., Quan, W. (eds.) GD 2007. LNCS, vol. 4875, pp. 291-302. Springer, Heidelberg (2008)

6. Eades, P., Feng, Q.W., Lin, X., Nagamochi, H.: Straight-line drawing algorithms for hierarchical graphs and clustered Graphs. Algorithmica 44, 1-32 (2006)

7. Feng, Q.W., Cohen, R.F., Eades, P.: Planarity for clustered graphs. In: Spirakis, P.G. (ed.) ESA 1995. LNCS, vol. 979, pp. 213-226. Springer, Heidelberg (1995)

8. Goodrich, M.T., Lueker, G.S., Sun, J.Z.: C-planarity of extrovert clustered graphs. In: Healy, P., Nikolov, N.S. (eds.) GD 2005. LNCS, vol. 3843, pp. 211-222. Springer, Heidelberg (2006)

9. Gutwenger, C., Jünger, M., Leipert, S., Mutzel, P., Percan, M., Weiskircher, R.: Advances in c-planarity testing of clustered graphs. In: Goodrich, M.T., Kobourov, S.G. (eds.) GD 2002. LNCS, vol. 2528, pp. 220-235. Springer, Heidelberg (2002)

10. Gutwenger, C., Jünger, M., Leipert, S., Mutzel, P., Percan, M., Weiskircher, R.: Subgraph induced planar connectivity augmentation. In: Bodlaender, H.L. (ed.) WG 2003. LNCS, vol. 2880, pp. 261-272. Springer, Heidelberg (2003)

11. Hopcroft, J., Tarjan, R.E.: Efficient planarity testing. J. ACM 21(4), 549-568 (1974)

12. Jelínková, E., Kára, J., Kratochvíl, J., Pergel, M., Suchý, O., Vyskočil, T.: Clustered planarity: Small clusters in eulerian graphs. In: Hong, S.-H., Nishizeki, T., Quan, W. (eds.) GD 2007. LNCS, vol. 4875, pp. 303-314. Springer, Heidelberg (2008) 